\title{
Theranostics
}

$2011 ; 1: 381-394$

Research Paper

\section{Theranostic cRGD-BioShuttle Constructs Containing Temozolomide- and Cy7 For NIR-Imaging and Therapy}

\author{
Manfred Wiessler ${ }^{1}$, Ute Hennrich ${ }^{1}$, Rüdiger Pipkorn², Waldemar Waldeck ${ }^{3}$, Liji Caoํ, Jörg Peter ${ }^{1}$, Volker \\ Ehemann ${ }^{4}$, Wolfhard Semmler ${ }^{1}$, Twan Lammers ${ }^{5}$, Klaus Braun ${ }^{1 凶}$ \\ 1. DKFZ, Dept. of Imaging and Radiooncology, INF 280, D-69120 Heidelberg, Germany \\ 2. DKFZ, Central Peptide Synthesis Unit, INF 580, D-69120 Heidelberg, Germany \\ 3. DKFZ, Div. of Biophysics of Macromolecules, INF 580, D-69120 Heidelberg, Germany \\ 4. University of Heidelberg, Institute of Pathology, INF 220, D-69120 Heidelberg, Germany \\ 5. RWTH Aachen University, Dept. of Experimental Molecular Imaging, Pauwelsstrasse 30, D-52074 Aachen, Germany
}

$\triangle$ Corresponding author: Klaus Braun, Ph.D., German Cancer Research Center (DKFZ), Dept. of Imaging and Radiooncology, Im Neuenheimer Feld 280, D-69120 Heidelberg, Germany. Phone: +49 6221-42 2495; Fax: +49 6221-42 3326; e-mail: k.braun@dkfz.de

(C) Ivyspring International Publisher. This is an open-access article distributed under the terms of the Creative Commons License (http://creativecommons.org/ licenses/by-nc-nd/3.0/). Reproduction is permitted for personal, noncommercial use, provided that the article is in whole, unmodified, and properly cited.

Received: 2011.05.18; Accepted: 2011.12.01; Published: 2011.12.11

\begin{abstract}
Innovative and personalized therapeutic approaches result from the identification and control of individual aberrantly expressed genes at the transcriptional and post-transcriptional level. Therefore, it is of high interest to establish diagnostic, therapeutic and theranostic strategies at these levels. In the present study, we used the Diels-Alder Reaction with inverse electron demand $\left(D A R_{\text {inv }}\right)$ click chemistry to prepare a series of cyclic RGD-BioShuttle constructs. These constructs carry the near-infrared (NIR) imaging agent Cy7 and the chemotherapeutic agent temozolomide (TMZ). We evaluated their uptake by and their efficacy against integrin $\alpha_{v} \beta_{3}$-expressing MCF7 human breast carcinoma cells. In addition, using a mouse phantom, we analyzed the suitability of this targeted theranostic agent for NIR optical imaging. We observed that the cyclic RGD-based carriers containing TMZ and/or Cy7 were effectively taken up by $\alpha_{v} \beta_{3}$-expressing cells, that they were more effective than free TMZ in inducing cell death, and that they could be quantitatively visualized using NIR fluorescence imaging. Therefore, these targeted theranostic agents are considered to be highly suitable systems for improving disease diagnosis and therapy.
\end{abstract}

Key words: BioShuttle, Click-Chemistry, inverse Diels Alder Reaction, Ligation chemistry, NIR Imaging, RGD, targeted Imaging, targeted Therapy, Temozolomide, Theranostics

\section{Introduction}

The rapid progress in genomic and proteomic research has contributed substantially to the development of novel and more specific diagnostics and therapeutics. Such advanced interventions facilitate individualized and personalized treatment regimens, and increasingly involve diagnostics and therapeutics based on antibodies, peptides and nucleic acids. In the field of diagnostics, for instance, peptides and antisense molecules coupled to contrast agents have been shown to be excellent tools for detecting aberrantly expressed receptors and mRNA in tumor cells using positron emission tomography [1-5], magnetic resonance imaging (MRI) [6-9], ultrasound (US) [11-13] and near-infrared fluorescence imaging (NIR) [14-17]. 
Both targeted therapeutics and molecular imaging agents have already been shown to be highly suitable materials for facilitating disease diagnosis and therapy. Therefore cell- and tissue-specific targeting with so-called theranostic agents, which combine diagnostic and therapeutic properties within a single formulation, are more and more considered to be one of the next major leaps forward for enabling personalized medicine [17-19]

With regard to this expanding field of theranostics, our considerations focused on receptor expression by tumor cells and tumor blood vessels. The expression of the $\alpha_{\mathrm{v}} \beta_{3}$ integrin receptor, for instance, is hardly detectable in normal (endothelial) cells, but on tumor blood vessels and (certain) tumor cells, it is highly overexpressed. This fact has already been shown to enable effective discrimination between the tumor and surrounding healthy tissue, as exemplified e.g. by studies in which cyclic RGD (cRGD) was coupled to PET, MRI, US and NIR imaging agents, such as ${ }^{18} \mathrm{~F},{ }^{68} \mathrm{Ga},{ }^{64} \mathrm{Cu}$, gadolinium, iron oxides, microbubbles, quantum dots and Cy5.5 [2, 3, 5, 6-8, 11, 13-16, 20].

The use of RGD peptides for cell- and tissue-targeting permits to achieve much higher local concentrations of active and imaging molecules at the pathological site compared to non-specific agents. Therefore, we here expand our modular peptide nucleic acid (PNA)-based BioShuttle delivery platform $[5,21-24]$ with a cyclic(RGDfK) molecule, targeted to the $\alpha_{v} \beta_{3}$ integrin receptor overexpressed by endothelial and (certain) cancer cells. We describe its functionalization with a tetramer containing the chemotherapeutic agent temozolomide (TMZ) and/or an NIR imaging agent based on Cy7. An advanced click chemistry-based approach, based on the Diels-Alder-Reaction with inverse electron demand $\left(\mathrm{DAR}_{\text {inv }}\right)$ [24-28] enabled the simple and straightforward functionalization of the cRGDfK-containing PNA-based carrier construct. Several different constructs were prepared (cRGD-TMZ4, cRGD-TMZ ${ }^{3}-\mathrm{Cy} 7^{1}, \quad$ cRGD-TMZ2-Cy72 and cRGD-Cy $7^{4}$ ) and they were evaluated in vitro with regard to cellular uptake and induction of cell death. In addition, the suitability of cRGD-Cy $7^{4}$ for NIR fluorescence imaging was evaluated in an appropriate animal phantom. The results show that cRGD-based carriers containing TMZ and/or Cy7 were effectively taken up by $\alpha_{\mathrm{v}} \beta_{3}$ integrin expressing MCF7 breast cancer cells, that they were more effective in inducing cell death than free TMZ, and that they could be quantitatively visualized in a XFM-2 mouse phantom.

\section{Material \& Methods}

\section{Cell culture}

Estrogen-sensitive MCF7 human adenocarcinoma breast cancer cells (DKFZ, Dept. B040) were used. They were cultured and maintained at $37^{\circ} \mathrm{C}$ in a $5 \%$ $\mathrm{CO}_{2}$ atmosphere in DMEM (Gibco, Germany), supplemented with $10 \%$ fetal calf serum (Biochrome, Germany).

\section{Synthesis of TMZ-tetrazine diene $(I)$ as reaction partner for $D A R_{\text {inv }}$}

Compound 1 (Figure 1) was synthesized by the reaction of 4-methyl-5-oxo-2,3,4,6,8-pentazabicyclo [4.3.0]nona-2,7,9-triene-9-carboxamide \{temozolomide - TMZ (Sigma-Aldrich, Germany)\} with $\mathrm{N}$-(2-aminopropyl)-4-(6-(pyrimidine-2-yl)-1,2,4,5-tetra zine-3-yl)benzamide [28]. This compound acts as the dienophile component in DAR inv.

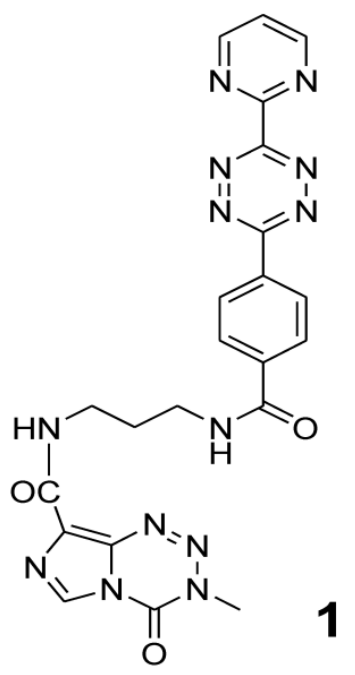

Figure I. Chemical structure of 4-methyl-5-oxo2,3,4,6,8-pentazabicyclo[4.3.0]nona-2,7,9-triene-9-carboxamide covalently attached to the $\varepsilon$-amino group of lysine which in turn is bound to the carboxamide group of $\mathrm{N}$-(2-aminopropyl)-4-(6-(pyrimidine-2-yl)-I,2,4,5-tetrazine-3-yl)b enzamide (I) [28].

\section{Synthesis of Reppe anhydride derivative (2) - corresponding $D A R_{\text {inv }}$ reaction partner}

The synthesis of tetracyclo-[5.4.21,7.02,6.08,11]3,5dioxo-4-aza-9,12-tridecadiene 2 was documented by Reppe [29]. The detailed chemical reaction steps to obtain the corresponding acetic acid chloride derivative 2 (Figure 2) are described in [30]. 


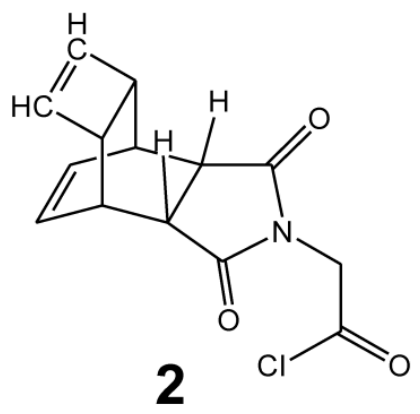

Figure 2. Chemical structure of the Reppe Anhydride 2-amino acetic acid (glycine) chloride derivative (2).

\section{Synthesis of the functionalized amide-monomer Reppe anhydride (3) for the backbone}

The synthesis of Fmoc-protected (2-aminoethyl)glycine (PNA backbone without nucleobase) was documented by Atherton and Sheppard [31]. The reaction product 3 is shown in Figure 3 and was prepared as described in [30].

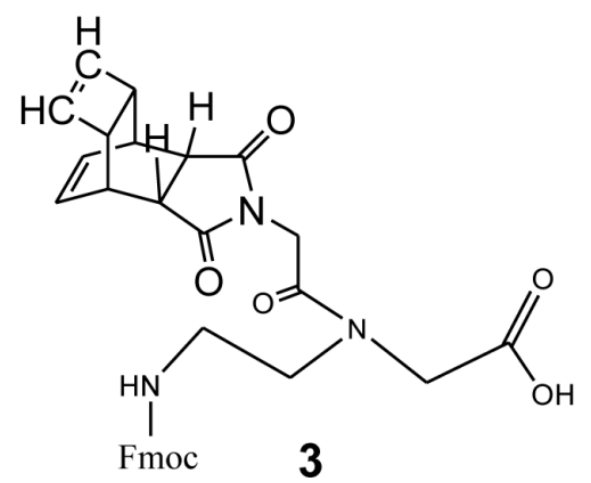

Figure 3. Chemical structure of Fmoc protected-[(2-aminoethyl)glycine] monomer $\mathrm{N}$-functionalized with the glycine derivative of Reppe Anhydride (3).

\section{Synthesis of the CRGD-amide tetramer func- tionalized with the Reppe anhydride (4)}

The synthesis of c(RGDfK)-tetra-(2aminoethyl)glycine peptide functionalized with the Reppe anhydride 3 was carried out by Fmoc-based solid phase synthesis. In a similar approach as published by Kubas et al. [32], all reaction steps were conducted on solid phase. The reaction scheme for the whole peptide synthesis is shown in Figure 4. All amino acid derivatives as well as the resin were obtained from Merck (Germany). The protected peptidyl resin was manually built on 2-chlorotrityl resin (1.5 $\mathrm{mmol} / \mathrm{g}, 0.1 \mathrm{mmol}$ scale). As the first amino acid, Fmoc-Asp-OAll was coupled to the resin in dichloromethane in the presence of $\mathrm{N}, \mathrm{N}$-diisopropylethylamine (DIPEA). After $16 \mathrm{~h}$ reaction time, free reaction sites on the resin were blocked (dichloromethane: methanol: DIPEA; 17:2:1, v/v/v, 3 $\times 30 \mathrm{~min}$ ). The other four Fmoc-protected amino acid derivatives [Fmoc-Gly-OH, Fmoc- $\mathrm{Arg}(\mathrm{Pbf})-\mathrm{OH}$, Fmoc-Lys(Dde)-OH and Fmoc-D-Phe-OH] were successively condensed using 2-(1H-benzotriazole1-yl)-1,1,3,3-tetramethyluronium hexafluorophosphate (HBTU) for activation in the presence of DIPEA in DMF, allowing a reaction time of 45 to $60 \mathrm{~min}$ for each amino acid. Fmoc-deprotection was performed by treatment of the resin with $50 \%(\mathrm{v} / \mathrm{v})$ piperidine-DMF for 2 and $5 \mathrm{~min}$. For the ring formation, the allyl protection group was cleaved using tetrakis(triphenylphosphine)-palladium (0.3 eq.) as a catalyst in dichloromethane containing morpholine (25 eq.). After $3 \mathrm{~h}$ reaction time, the resin was washed first with $5 \%$ DIPEA in DMF $(5 \times 5 \mathrm{~min})$ and then with $0.1 \%$ sodium diethyldithiocarbamate in DMF (5 $\times 5$ min). After Fmoc deprotection of the N-terminal amino acid, ring closure was conducted using 6-chlorohydroxybenzotriazole (PyCLOCK) (5 eq.) and DIPEA (5 eq.) in DMF for $17 \mathrm{~h}$. For the conjugation of the Reppe Anhydride containing amino acids, the $\varepsilon$-amino function of Lys was selectively deprotected by treatment of the resin with $2 \%$ hydrazine monohydrate in DMF for $3 \mathrm{~h}$. To the Lys residue the Fmoc protected diene derivatives were successively condensed four times as described above and the $\mathrm{N}$ terminal acid was acetylated. Cleavage from the resin as well as side-chain deprotection of the amino acid derivatives was achieved by treatment with $95 \%$ TFA, $2.5 \%$ TIS and $2.5 \%$ water for $4 \mathrm{~h}$. Purification of 4 was performed by semi-preparative reversed-phase HPLC (Dionex (Idstein, Germany): Ultimate 3000 LPG-3400A pump and variable four wavelength Ultimate 3000 VWD-3400RS UV/VIS detector $(222 \mathrm{~nm}$, $254 \mathrm{~nm}, 280 \mathrm{~nm}$ ); column: Chromolith Performance RP-18e column $(100 \times 10 \mathrm{~mm}$; Merck, Darmstadt, Germany). The solvent gradient was raised from $5 \%$ to $100 \%$ acetonitrile in $5 \mathrm{~min}$ at a flow rate of 6 $\mathrm{mL} / \mathrm{min}$. The aqueous phase consisted of water containing $0.1 \%$ TFA. $\left.t_{R}=3.58 \mathrm{~min}\right)$. The identity of the reaction product 4 (Figure 5A) was confirmed by MALDI-TOF-MS (m/z calculated for $\mathrm{C}_{101} \mathrm{H}_{119} \mathrm{~N}_{21} \mathrm{O}_{24}$ $\left([\mathrm{M}+\mathrm{H}]^{+}\right): 2012.15$ found 2012.1) (Figure 5C). MALDI-TOF-MS was performed on a Microflex ${ }^{\mathrm{TM}}$ LT (Bruker Daltonics, Bremen, Germany). 
A)

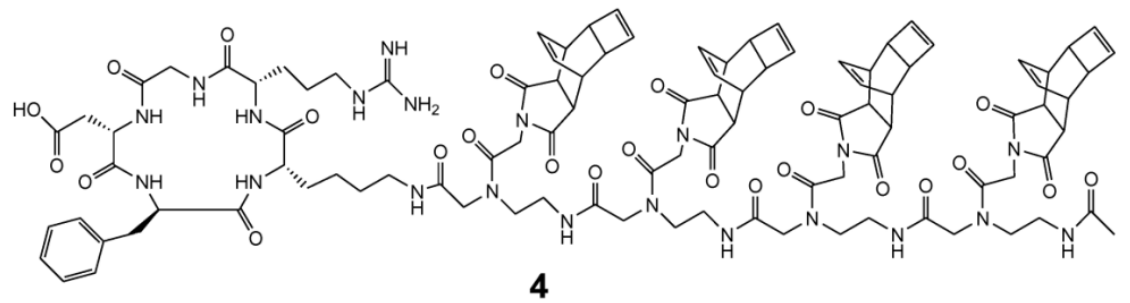

B)

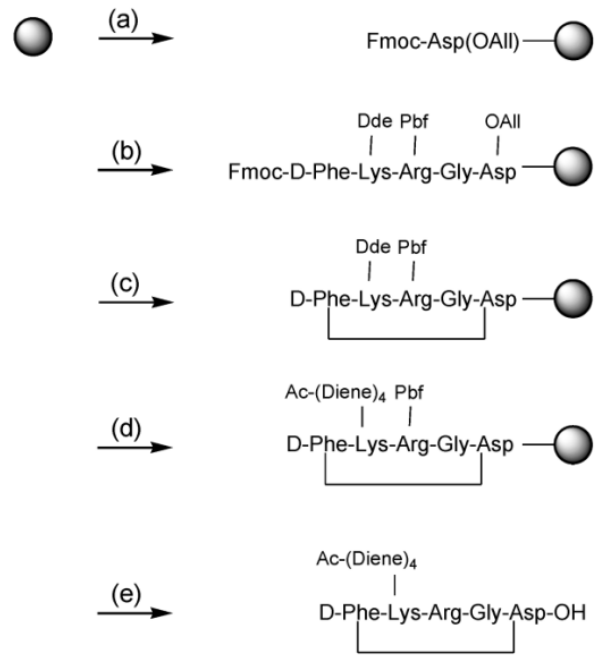

C)
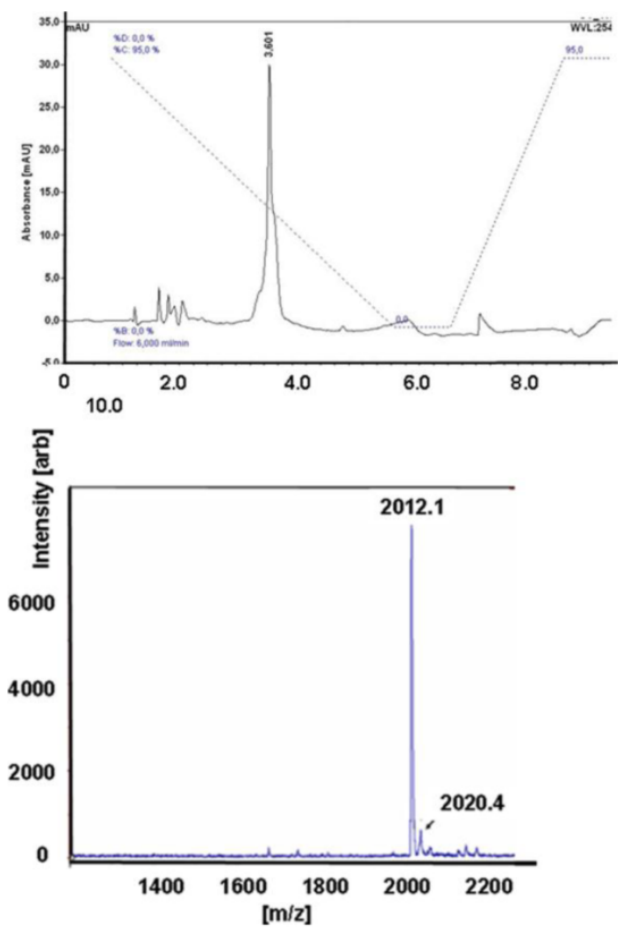

Figure 4. A) Shows the chemical structure of 4, the cRGD-peptide derivatized with tetra-[(2-aminoethyl)glycine] N-functionalized Reppe Anhydride. B) Synthetic route for the preparation of 4 . The solid phase support is depicted by the grey circle. (a) condensation of the first amino acid; (b) stepwise elongation on 2-chlorotrityl resin; (c) cyclization steps: I. Pd(PPh3)4, morpholine; 2. PyCLOCK, DIPEA; (d) conjugation to Reppe Anhydride derivative: I. Hydrazine monohydrate; 2. Stepwise elongation, 3. Acetylation; (e) cleavage from resin and deprotection: TFA, TIS, water. C) The upper panel shows the HPLC chromatogram of the purified peptide 4. The solvent gradient was raised from $5 \%$ to $100 \%$ acetonitrile in $5 \mathrm{~min}$ (starting immediately) at a flow rate of $6 \mathrm{ml} / \mathrm{min}$. The aqueous phase consisted of water containing $0.1 \%$ TFA. The lower panel shows the MALDI-TOF spectrum of the purified peptide 4. 
A)

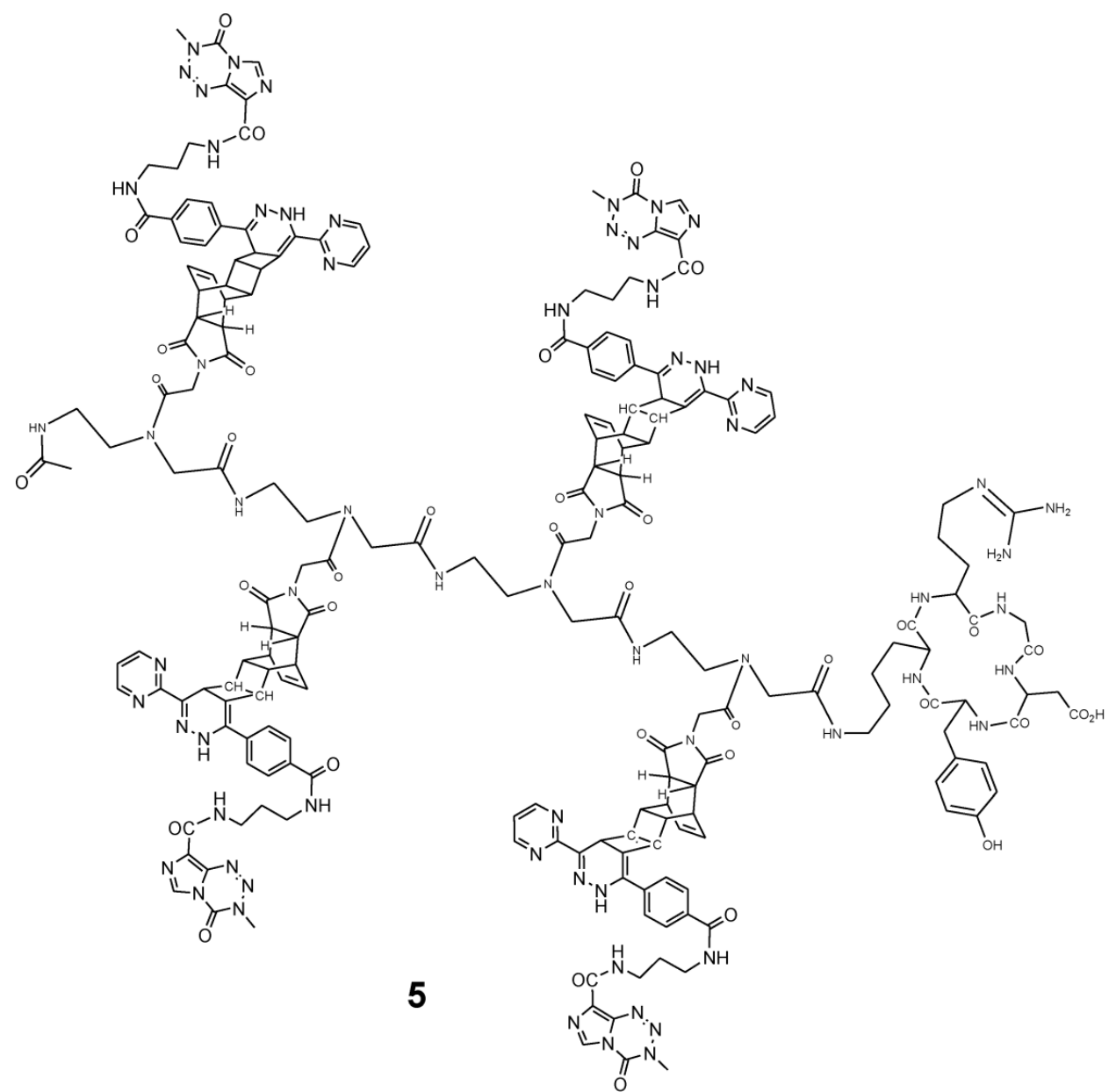

B)

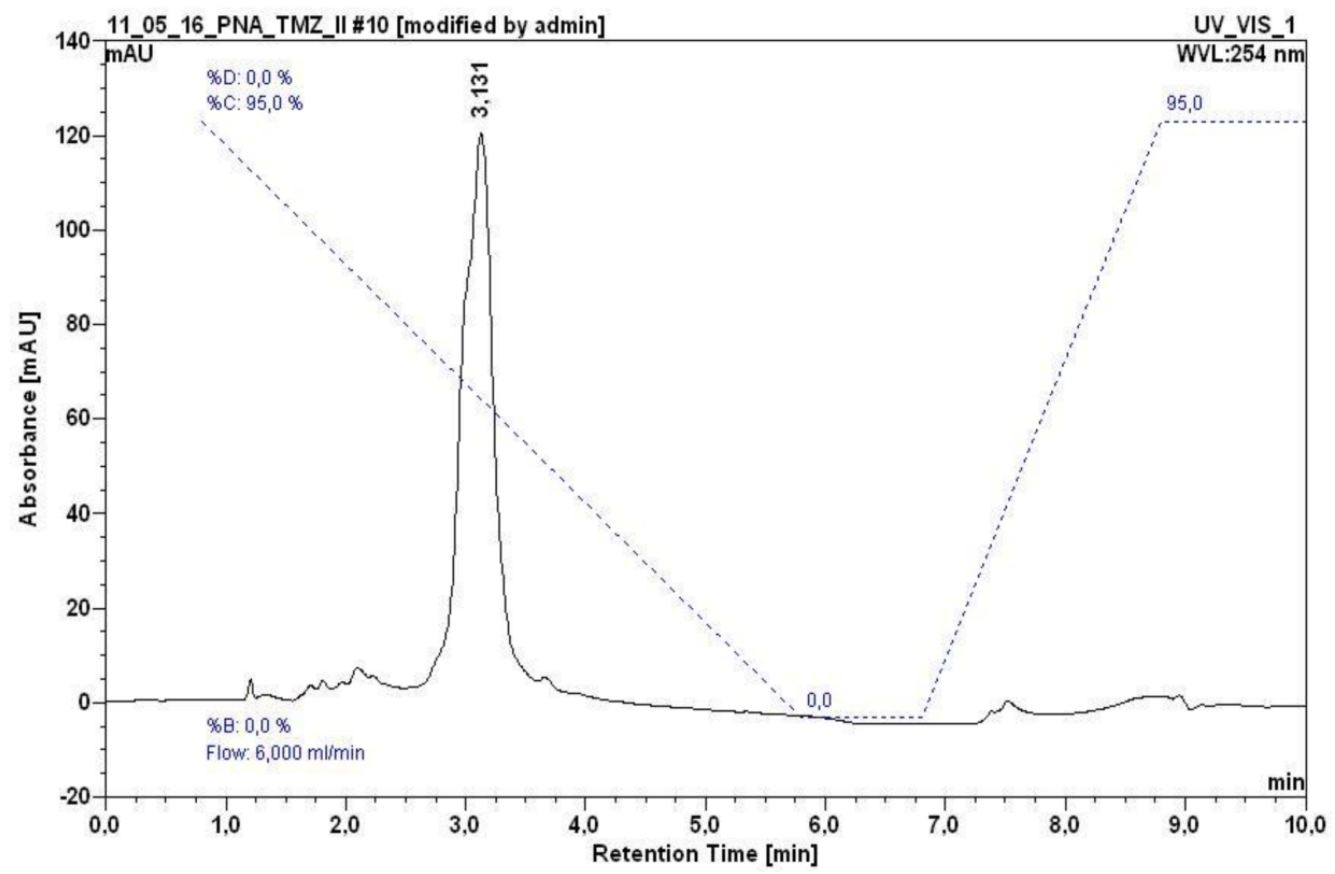

Figure 5. A) Chemical structure of the reaction product 5 (described as variant I, Table I) after ligation of 4 and I by use of the DARinv. B) HPLC chromatogram of purified 5. For HPLC conditions see Figure 4C. 


\section{Synthesis of CRGD-TMZ ${ }^{4}(5)$}

In order to realize sufficiently high local concentrations to kill target cells, such as $\alpha_{\mathrm{v}} \beta_{3}$ integrin expressing MCF7 cells, a targeted TMZ-conjugate with similarities to the previously documented cRGD-BioShuttle-TMZ was designed and synthesized [27]. Here, with the aim of enhancing the toxic effects against target cells, we connected the cRGD targeting peptide to a tetramer of PNA amide backbone-based building blocks functionalized with TMZ. The chemical synthesis of the $\mathrm{CRGD}-\mathrm{TMZ}^{4}$ (5) was carried out by the reaction of peptide 4 with 1 in the $D_{A R}$ inv according to the protocol described in [27, 30]. The compound was purified by semi-preparative HPLC (system and conditions see above, $t_{R}=3.13 \mathrm{~min}$ ). Its structure as well as the HPLC chromatogram are shown in Figure 5.

\section{Synthesis of Cy7 functionalized with a diaryl diene component (6)}

The ligation of Cy7 with $N$-(2-aminopropyl)-4-(6(pyrimidine-2-yl)-1,2,4,5-tetrazine-3-yl)benzamide was performed as described in [28].

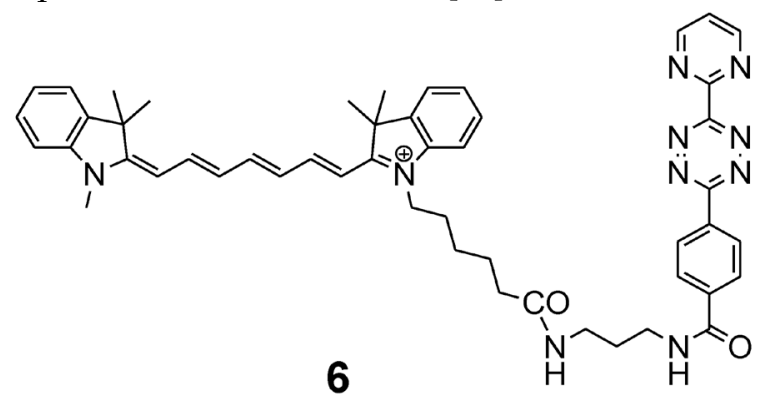

\section{Synthesis of CRGD-TMZ ${ }^{3}-\mathrm{Cy} 7^{1}$, CRGD-TMZ ${ }^{2}-\mathrm{Cy}^{2}$, and $\mathrm{CRGD}-\mathrm{Cy}^{4}$}

In order to evaluate the dose-dependent sensitivity of Cy7 in NIR imaging as well as the TMZ mediated treatment response, we designed the following variants based on the cRGD-Lys-PNA-like amide-tetramer 4 by use of the $\mathrm{DAR}_{\text {inv }}$ with Cy7-tetrazine diene 6 and TMZ-tetrazine diene 1, respectively (Table 1). 1 and 6 each react as the diene components whereas the Reppe anhydride in $\mathbf{4}$ functions as the dienophile reaction partner. Variant II possesses one Cy7 and three TMZ, variant III two Cy7 and two $\mathrm{TMZ}$, and variant IV four Cy7. By reaction of $\mathbf{4}$ with equimolar amounts of $\mathbf{1}$ and 6, respectively, a mixture of II and III was obtained which could be separated by semi-preparative HPLC (system and conditions see above; $\left.\mathrm{t}_{\mathrm{R}}(\mathbf{I I})=3.63 \mathrm{~min}, \mathrm{t}_{\mathrm{R}}(\mathbf{I I I})=3.95 \mathrm{~min}\right)$. The HPLC chromatograms of II, III, and IV are shown in Figure 7. The identity of the compounds was confirmed by ESI-MS.

Table I. Overview of the CRGD-TMZ-Cy7 conjugates (I-IV) used in this study:

\begin{tabular}{llll}
\hline & cRGD variants & TMZ & CY7 \\
\hline I & cRGD-TMZ $^{4}$ & $\mathbf{4}$ & - \\
II & cRGD-TMZ3-Cy71 $^{3}$ & $\mathbf{3}$ & $\mathbf{1}$ \\
III & cRGD-TMZ2-Cy72 $^{2}$ & $\mathbf{2}$ & $\mathbf{2}$ \\
IV & cRGD-Cy7 & - & $\mathbf{4}$ \\
\hline
\end{tabular}

Figure 6. Chemical structure of the indotricarbocyanine dye Cy7-tetrazine diene component $\mathbf{6}$ suitable for ligation by DAR inv with 4.
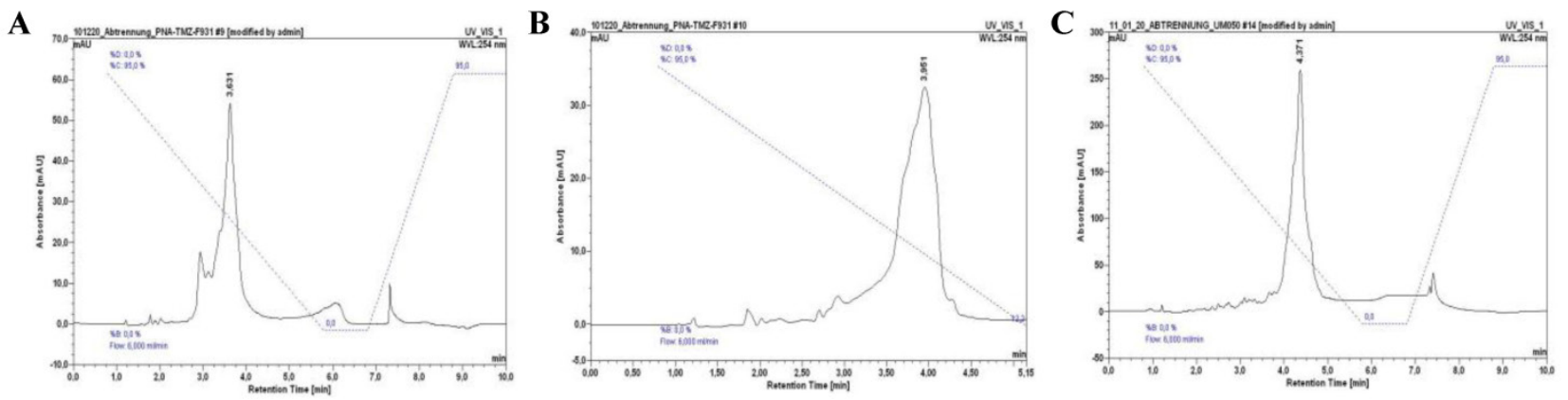

Figure 7. HPLC chromatograms of II (A), III (B) and IV (C). See Figure 4C for HPLC conditions. 


\section{NIR Fluorescence measurements of the Cy7 de- rivatives}

In order to characterize the fluorescence properties of the Cy7 containing constructs, excitation and emission measurements were performed. On the basis of the stock solution (1 $\mathrm{g}$ substance / L), the following dilution series was prepared: 1:10, 1:50, and 1:500. The corresponding emission intensities of the $\mathrm{Cy} 7$ derivatives were measured in the spectral range between 400 and $800 \mathrm{~nm}$. The following Cy7 derivatives were measured:

- $\quad$ cRGD-Cy74 (IV, Table 1)

- cRGD-TMZ $^{3}-$ Cy71 $^{1}$ (II, Table 1)

- the Cy7-tetrazine diene component 6 alone was investigated under identical conditions

\section{Confocal Laser scanning microscopy (CLSM)}

In order to reconfirm our data documenting the internalization of the cRGD into the cytoplasm of MCF-7 breast cancer cells we used the confocal laser scanning microscope (CLSM) of the Microscopy Core Facility of the German Cancer Research Center for qualified verification of the data. The pictures were taken with a Leica confocal microscope TCS SP5 II (excitation at $740 \mathrm{~nm}$, emission at $780 \mathrm{~nm}$ ) and examined with the Leica LAS-Software. 24 hours before CLSM measurements the MCF-7 cells $\left(5 \times 10^{5}\right)$, were cultivated in 8-well cell culture plates $\left(\mathrm{Lab}^{\mathrm{T}} \mathrm{Tek}^{\circledR}\right)$ and treated with cRGD-TMZ2-Cy72 (III, Table 1) $(3.7,11$, 33, and $100 \mu \mathrm{M}$ ) (Figure 11).

\section{Mouse phantom study}

A phantom study [XFM-2 Fluorescent Mouse phantom (Xenogen Corp)] was performed to assess the optical properties of cRGD-CY74 (IV) and cRGD-TMZ ${ }^{3}-\mathrm{Cy}^{1}$ (II) for its potential use in small animal imaging. As the probe had an absorption maximum at $745 \mathrm{~nm}$ (Figure 8), it was excited with a $733 \mathrm{~nm}$ diode laser $(20 \mathrm{~mW})$ equipped with an additional $740 \mathrm{~nm}-13$ band-pass filter, to limit the bandwidth of excitation light. Emitted light was detected with a Hamamatsu ORCA ER camera $\left(-45^{\circ} \mathrm{C}\right.$, high gain, no binning, lens F-number 2.8), equipped with a $786 \mathrm{~nm}-22$ band-pass filter to filter out the excitation light. The concentration of the probes was 1, 10 and $100 \mathrm{nM}$, respectively, and the measurements were performed in capillaries either in the mouse phantom or without the mouse phantom.

\section{Treatment of MCF7 breast cancer cells}

TMZ, cRGD-TMZ ${ }^{4}$ (I), and cRGD-TMZ2-Cy72 (III) were dissolved in $10 \%$ aqueous solution. MCF7 cells were grown as subconfluent monolayers in DMEM (control) and in DMEM containing appropri- ate amounts of TMZ, cRGD-TMZ ${ }^{4}$ and cRGD-TMZ2-Cy72, respectively, (100, 33, 11, and 3.7 $\mu \mathrm{M})$, and their effects were analyzed $48 \mathrm{~h}$ after treatment.

\section{Multiparametric flow cytometry analysis}

Experiments were performed using a Galaxy Pro flow cytometer (PARTEC, Germany), equipped with a $488 \mathrm{~nm}$ laser and $635 \mathrm{~nm}$ laser diode, with filter combination for Cy7. The use of the flow cytometry parameters forward (FSC) and sideward (SSC) scatter of the cells give an indication on drug effects through the relative cell size and structural effects such as granularity.

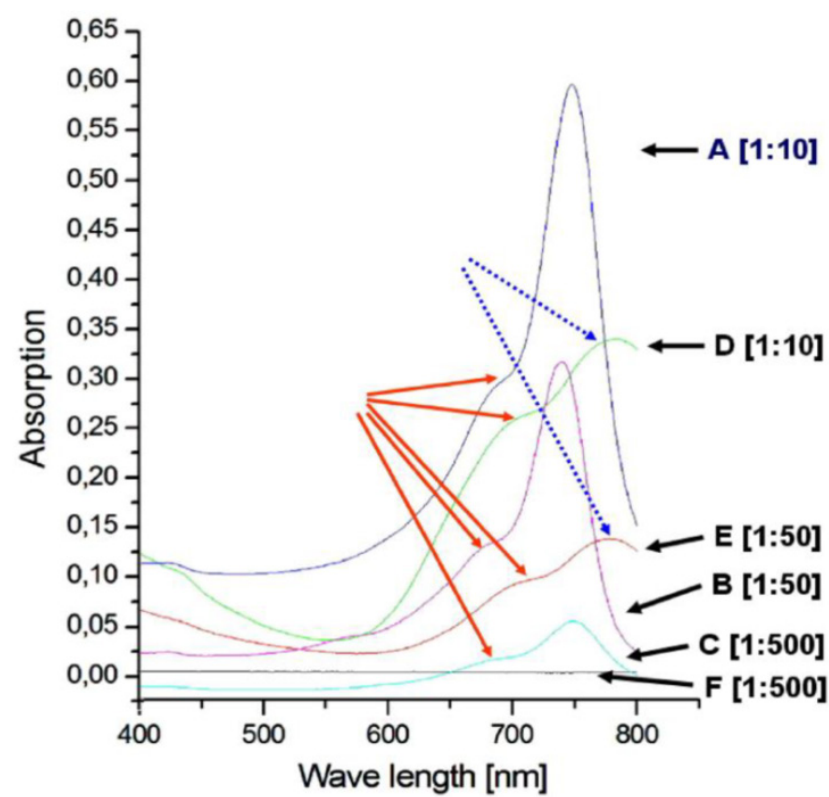

Figure 8. The diagram shows the absorption spectra of the Cy7 tetrazine diene component 6 (A, B, C) and cRGD-TMZ3-Cy7। II (D, E, F). A) Dark blue: 6: dilution: I:I0; B) Magenta: 6: dilution I:50; C) Blue: 6: dilution I:500; D) Green: II: dilution I: I 0; E) Dark brown: II: dilution I:50; F) Dark gray: II: dilution I:500; The red arrows point to the shoulders $(710-740 \mathrm{~nm})$. The dashed blue arrows point to the maxima of $D$ and $E$ shifted on the wavelength axis towards $800 \mathrm{~nm}$.

\section{Results}

\section{Absorption spectra}

First, we characterized the absorption spectra of the synthesized $\mathrm{Cy} 7$ fluorescent diene component $\mathbf{6}$, which is ready for $D_{A R}$ inv. As depicted in Figure 8 A-C, the measurements showed a maximum at 745 $\mathrm{nm}$ and a shoulder at 710 and $740 \mathrm{~nm}$, dependent on the concentration of the measured solutions. We observed decreasing absorption magnitudes $(0.6,0.3$, 0.05 ) in relation to the dilution from 1:10, 1:50, to $1: 500$ 
of the Cy7-tetrazine diene component 6 . As exemplified by the curves in Figure 8 D-E, the absorption measurements of the reaction product cRGD-TMZ3 $-C Y 7^{1}$ (after DAR ${ }_{\text {inv }}$ ligation) showed a shift of the maximum to $\sim 800 \mathrm{~nm}$ and a similar dilution-dependent decrease in absorption height $(0.325$ and 0.125 ; for 1:10 and 1:50 dilutions, respectively). At a dilution of 1:500 no maximum absorption peak was observed for cRGD-TMZ3-Cy7.

\section{Phantom study}

The cRGD-Cy7 ${ }^{4}$ conjugate (IV, Table 1 ) was designed as a prototype to investigate NIR efficiency parameters, like refinement of spatial resolution and sensitivity of the NIR methodology. In order to assess the optical properties of cRGD-TMZ ${ }^{3}-\mathrm{Cy}^{71}$ (II) and
cRGD-Cy74 (IV) for its potential use in preclinical in vivo imaging, two experimental phantom studies were performed. The first set of experiments focused on capillaries [cRGD-TMZ3-Cy71 (II, Table 1) and cRGD-Cy $7^{4}$ (IV, Table 1)] placed in direct view of the camera. This setup was chosen to suppress photon scattering and absorption, and served to quantitatively detect the yield of emitted light related to probe concentration. Data were obtained for three different probe concentrations, and integrated intensity values were calculated in the indicated ROIs of the projection images. As shown in Figure 9, the observed values correlated linearly to the probe concentrations. The fluorescence intensities of IV (cRGD-Cy $7^{4}$ ) are ten times higher as measured in II (cRGD-TMZ3 -Cy71).

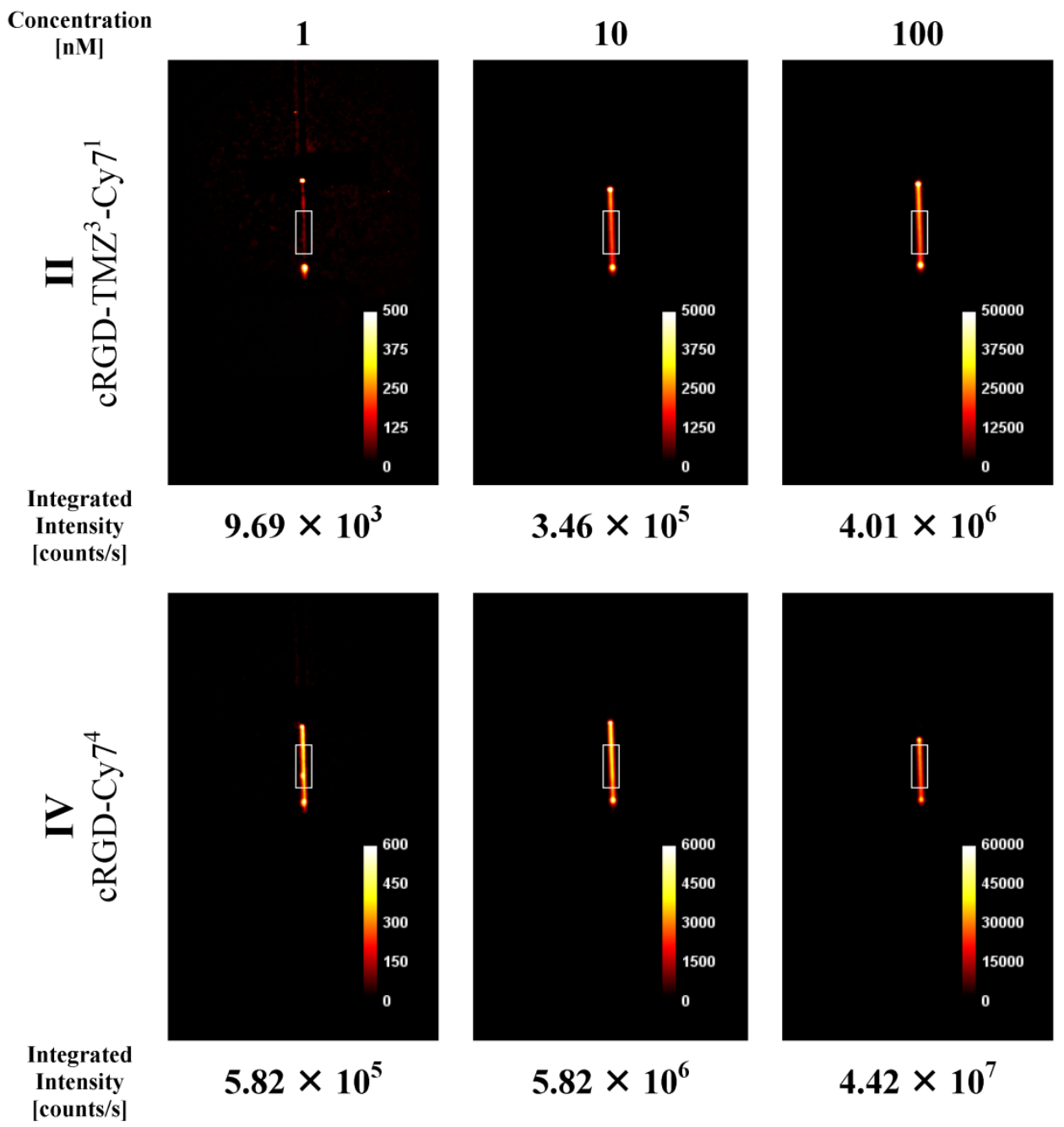

Figure 9. NIR intensity data acquisition in $0.9 \mathrm{~mm}$ capillary sources filled with three different concentrations of the $c R G D-T M Z^{3}-C y 71$ (II) and the cRGD-Cy 74 (IV). Counts are normalized for exposure time. 

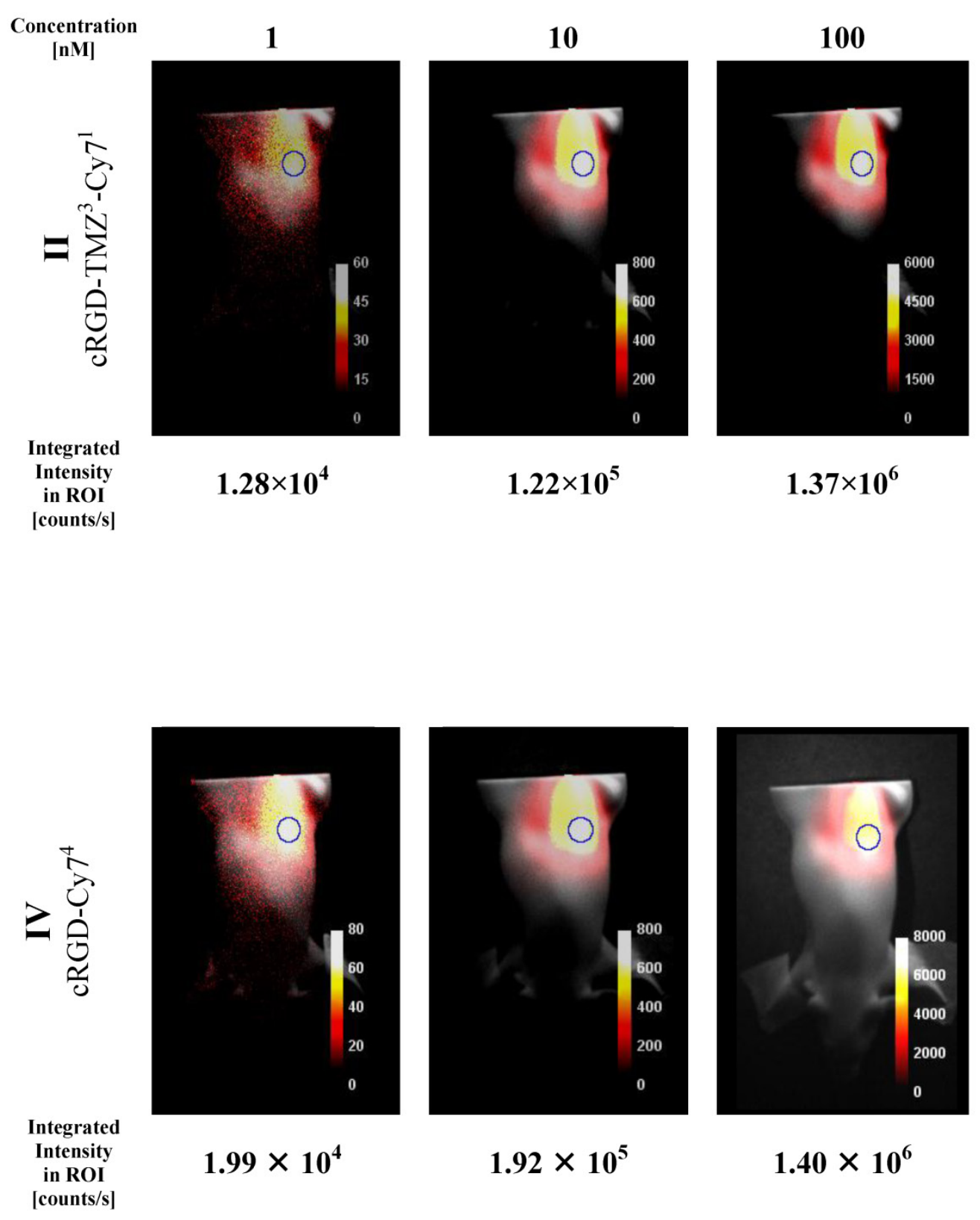

Figure 10. NIR intensity data acquisition of three different quantities of cRGD-Cy 74 (IV) placed inside rod opening A of the $X F M-2$ Fluorescent Mouse phantom (Xenogen Corp.). Counts are normalized for exposure time. Integrated intensity values are taken from the ROls as indicated.

In a second set of imaging experiments we investigated the XFM-2 Fluorescent Mouse phantom (Xenogen Corp.). This phantom approximates the optical properties of tissues, such as scattering and light attenuation with respect to the corresponding wavelength range. As shown in Figure 10, the Cy7 signal was detected at concentration levels that are commonly used in animal studies. It is evident that the fluorescence intensities of II (cRGD-TMZ3-Cy71) in the mouse phantom differ only slightly from IV (cRGD-Cy74) with four Cy7 fluorophores per mole- cule. Importantly, in line with the results obtained in the capillary experiment (Figure 9) the signal intensities correlated linearly with the concentration of the probe.

\section{Cellular localization of Cy7 using Confocal Laser Scanning Microscopy (CLSM)}

In order to investigate the cellular localization of cRGD-TMZ ${ }^{4}$ (I, Table 1), we designed a variant carrying two Cy7 moieties and two TMZ moieties (cRGD-TMZ ${ }^{2}-\mathrm{Cy}^{2}$, III, Table 1). This probe was applied to the culture media of $\mathrm{a}_{\mathrm{v}} \beta_{3}$ integrin-expressing 
MCF7 cells. As shown in Figure 11 after $48 \mathrm{~h}$ of incubation with III, a clear perinuclear Cy7 fluorescence signal was observed. Line $\alpha$ of Figure 11 represents the merged pictures of $\beta$ (DIC) and $\gamma$ (fluorescence). At a concentration of $33 \mu \mathrm{M}$, damage of cellular structures was evident. This observation was confirmed at concentrations of $100 \mu \mathrm{M}$, where no morphologically intact cells were visible. This can be clearly seen in the DIC pictures in Line $\beta$ : at a con-

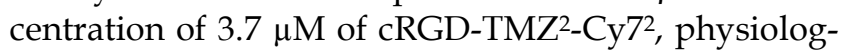

ically intact structures could still be observed. At 11 $\mu \mathrm{M}$, cellular structures were adelomorphic, and at a concentration of $33 \mu \mathrm{M}$, solely disintegrated cell mass was observed. It is interesting to note that at this concentration the cellular structures were still visible in the fluorescence images (Line $\gamma$ ), but absent in DIC. The cRGD-TMZ2-Cy72 appears to shrink the MCF7 cells. Upon treatment with a concentration of $100 \mu \mathrm{M}$, no morphologically intact cell structures could be observed any more.

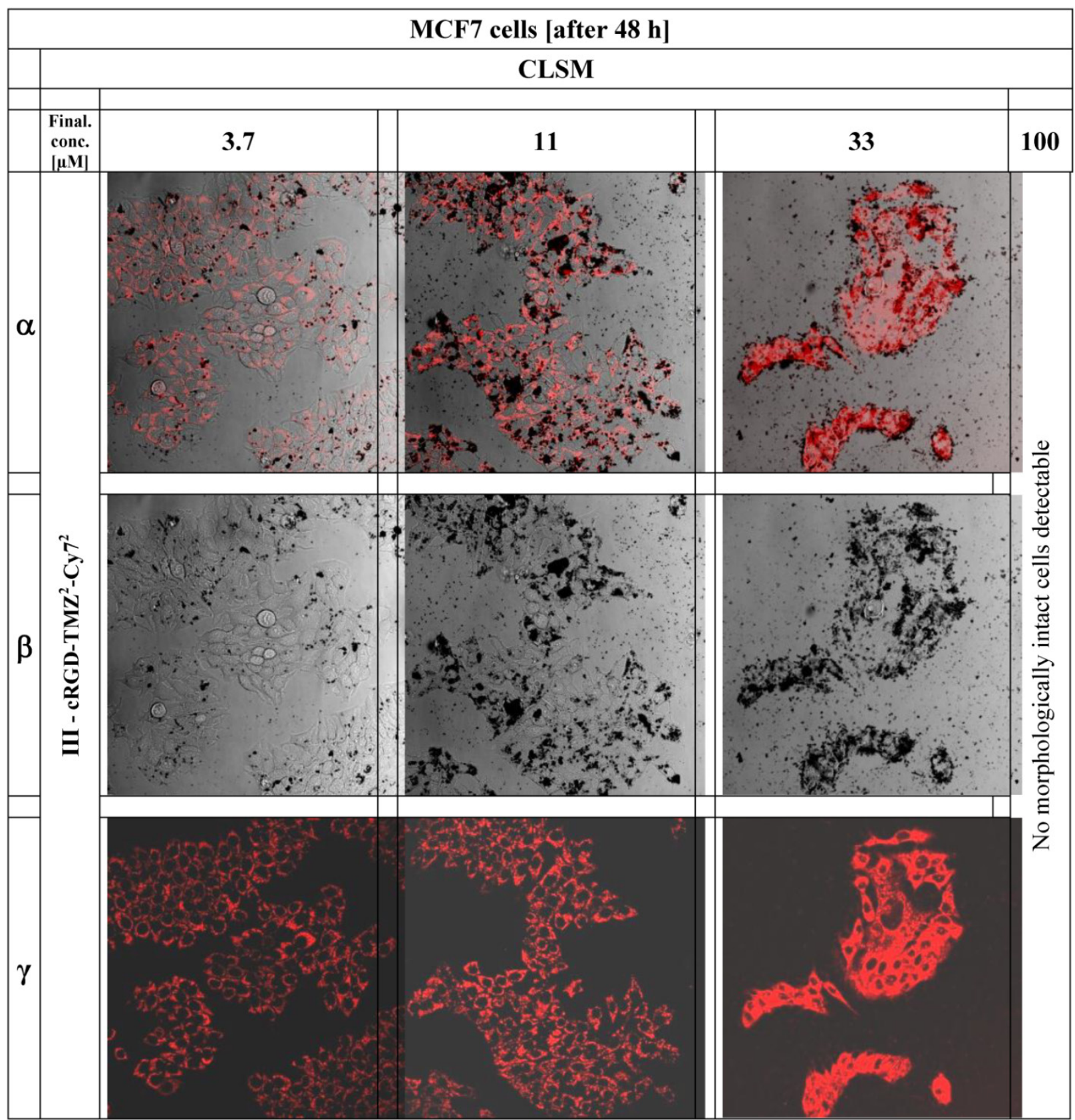

Figure II. The figure shows CLSM pictures of MCF7 breast cancer cells after application of cRGD-TMZ2-Cy72 (III, Table I). The columns of the figure illustrate the increasing concentrations of cRGD-TMZ2-Cy72 from left to right: $3.7,1$ I, 33, and I00 $\mu M$. 


\section{Multiparametric FACS analysis}

To further investigate the effects of free and cRGD-targeted TMZ on the structures of MCF7 cells, multiparametric flow cytometry was performed (Figure 12). We tested two major parameters: R1 represents the relative amount of cells (counts), and R2 represents the granularity, which is influenced by the morphology of the cell, the structure of the nuclei and the quantity of cytoplasmic compartments (such as mitochondria, Golgi, etc). The morphological parameters of the FACS analysis revealed an almost unaltered cell size in untreated cells (line 1), as well as in cells which were treated with free TMZ at a concentration of $33 \mu \mathrm{M}$ (line 2). In contrast to this, the gran- ularity changed and showed an increased fraction of more granulized cells upon treatment with cRGD-TMZ2_-Cy72 (III, Table 1). The MCF7 cell fraction with the highest amount of granularity $(29.4 \%)$ appeared after treatment at a concentration of $33 \mu \mathrm{M}$. At a concentration of $100 \mu \mathrm{M}$, no morphologically intact cells could be detected, which is in line with the results presented in Figure 11. Also in line with the results in Figure 11, when using FACS to analyze Cy7 uptake, it was found that cRGD-TMZ2-Cy72 was effectively taken up by MCF7 cells (Figure 12, line 3-5). Increased relative fluorescence intensity for cRGD-TMZ ${ }^{2}-\mathrm{Cy}^{2}$ could be detected, too. Both parameters suffice for a rough cell characterization.

\begin{tabular}{|c|c|c|c|c|c|c|c|}
\hline & \multicolumn{3}{|c|}{ Fin. Conc. $[\mu \mathrm{M}]$} & \multicolumn{2}{|c|}{ MCF-cells } & \multirow[b]{2}{*}{$\begin{array}{c}\text { R1 } \\
{[\%]}\end{array}$} & \multirow[b]{2}{*}{$\begin{array}{c}\mathbf{R 2} \\
{[\%]}\end{array}$} \\
\hline & & & & Granularity & Cy7 fluorescence & & \\
\hline 1 & نे & & & 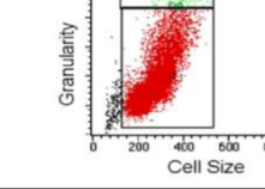 & Fluorescence Intensity & - & - \\
\hline 2 & $\sum_{i}^{N}$ & 33 &. & cell Size & 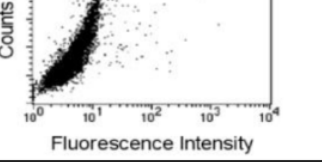 & 99.1 & 0.9 \\
\hline 3 & $\underset{7}{7}$ & 3.7 & 产 & 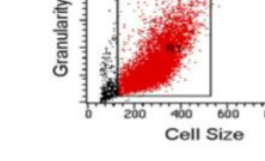 & 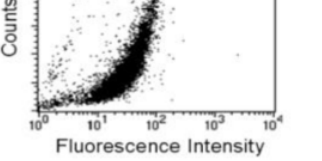 & 98.6 & 1.4 \\
\hline 4 & 主 & 11 & & 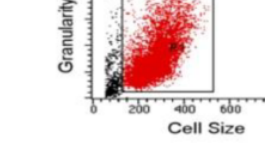 & 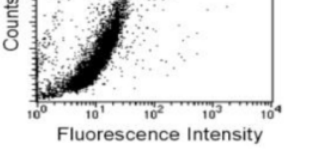 & 97.2 & 2.8 \\
\hline 5 & $\Xi$ & 33 & & 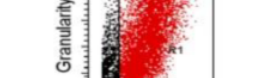 & 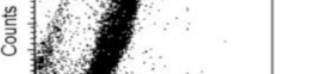 & 70.6 & 29.4 \\
\hline 6 & & 100 & & No morphologi & tact cells detectable & & \\
\hline
\end{tabular}

Figure 12. FACS analysis demonstrating the effects of free TMZ and different concentrations of cRGD-TMZ2-Cy 72 (III, Table I) on cell granularity and on cell size. It can be seen that cell size (RI) and granularity (R2) barely differed between untreated control cells and cells treated with free TMZ. For cRGD-TMZ2-Cy72, cell granularity increased with increasing dose. Upon incubation with $100 \mu M$ of CRGD-TMZ2-Cy72, no cells survived, and the detection of morphological changes was not possible. 


\section{Discussion}

Significant advances in genetics and proteomics in the past two decades resulted in a paradigm shift in (anticancer) drug therapy, from standard (mostly low-molecular-weight) chemotherapeutic drugs, to molecularly targeted therapeutic interventions. In parallel, molecular information and targeting strategies also started to influence the diagnostic field, giving rise to more disease-specific diagnostic agents and to molecular imaging techniques. The term molecular imaging was introduced approximately ten years ago, and is used to describe the diagnostic field which focuses on the analysis of biological processes at the molecular level, including e.g. receptor expression and enzyme activity [33]. Molecular imaging is considered as a pivotal technology for facilitating targeted (and personalized) therapy, enabling e.g. the distinction of malignant tissue from surrounding healthy tissue, the staging of tumors as well as the selection of therapeutics on the basis of receptor expression, and the visualization and staging of (micro)metastases.

In recent years, more and more attempts were made to combine (molecular) imaging and (targeted) therapy, giving rise to the highly innovative and highly interdisciplinary field of theranostics [17-20, 34]. Having these efforts in mind, we prepared an $\alpha_{v} \beta_{3}$ integrin targeted BioShuttle-based carrier material [5, 21-24, 35], consisting of amide-based building blocks whose backbone originates from peptide nucleic acids (PNA) [36-40]. We functionalized this BioShuttle with the alkylating chemotherapeutic agent temozolomide (TMZ) and the NIR dye indotricarbocyanine (Cy7). We previously showed that the cytotoxic effects of TMZ connected to a BioShuttle-based carrier material were increased through elevation of the local TMZ concentration at the surface of and inside $\alpha_{v} \beta_{3}$ integrin expressing cancer cells as compared to free TMZ [27]. Encouraged by these initial results, we here extended our efforts by synthesizing variants of this construct, in which the PNA-based and cRGD-targeted carrier moiety was functionalized with different amounts of TMZ and Cy7. Cellular granularity, which is known to be an adequate FACS parameter for assessing the morphological changes of cells upon exposure to cytotoxic agents [41], was used to evaluate the effect of (targeted) TMZ treatment on the induction of cell death. As expected, an increase in the granularity of MCF7 cells was observed upon exposing them to increasing concentrations of cRGD-targeted TMZ. This finding was corroborated by the fact that no morphologically intact MCF7 cells could be observed us- ing CLSM after exposing them to high concentrations of this agent. These results show that cRGD-TMZ2-Cy72 is (much) more potent as a cytotoxic agent compared to TMZ alone, which may be due on the one hand to the $\alpha_{v} \beta_{3}$ targeting cRGD moiety and on the other hand to the incorporation of two TMZ moieties.

To visualize the cRGD-targeted TMZ-containing constructs, the NIR dye Cy7 was incorporated. It is interesting to note that the absorption measurements of the Cy7-tetrazine precursor 6 , the reaction partner for inverse Diels-Alder (DAR inv)-based click chemistry, revealed an absorption maximum of $745 \mathrm{~nm}$ and that this maximum shifted to approximately $800 \mathrm{~nm}$ after ligation to the carrier material. To what extent fluorescence resonance energy transfer effects are involved in the shift of the absorption maximum toward $800 \mathrm{~nm}$ remains to be clarified. Analogous NIR imaging studies with Cy7-labeled neutrophil-specific agents showed no differences in spectra between non-conjugated and peptide-conjugated $\mathrm{Cy} 7$ dyes [42]. The fact that the Cy7 precursor used here is ligated to a diaryl-tetrazine, acting as a diene reaction partner for the $\mathrm{DAR}_{\text {inv, }}$ might explain this shift, but further experiments are required to confirm this assumption. Using capillaries and the XFM-2 phantom, which simulates the scattering and light attenuation of tissues in mice, we finally confirmed that the prepared Cy7-containing constructs are highly suited for NIR fluorescence imaging. Strong signals were observed even at a dose of $1 \mathrm{nM}$, and signal intensities linearly correlated with the concentrations of the probes. Remarkable is the fact that the use of probe II with one $\mathrm{Cy} 7$ as an imaging molecule (cRGD-TMZ ${ }^{3}-\mathrm{Cy}^{1}$ ) is sufficient for imaging as observed in the mouse phantom studies. The measurements of construct IV containing four Cy7 molecules (cRGD-Cy $\left.7^{4}\right)$ did not show much higher fluorescence intensities. This offers the possibility of conjugating other imaging or therapeutic probes to the versatile BioShuttle backbone, e.g. a chelator for radiometal complexation and/or an additional drug, in order to obtain multimodal theranostic formulations for simultaneously delivering multiple chemotherapeutic drugs to cancer cells. The results obtained here provide some initial experimental evidence in this direc-

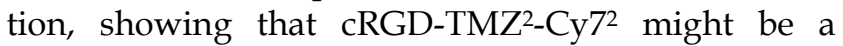
promising agent for targeted therapy combined with optical imaging of tumors. Experiments to validate these findings in living mice, as well as studies to evaluate the in vivo antitumor efficacy of these integrin-targeted theranostic agents, are planned for the near future. 


\section{Conclusion}

Using the inverse Diels-Alder reaction, several different BioShuttle-PNA constructs targeted to $\alpha_{v} \beta_{3}$ integrins and functionalised with TMZ as a therapeutic agents and with $\mathrm{Cy} 7$ as an imaging agent were prepared. One of these compounds, i.e cRGD-TMZ2-Cy72, showed enhanced cytotoxicity as compared to free TMZ, as well as very favourable imaging properties in NIR measurements. These results provide initial proof-of-principle for the potential of this formulation to be used as a theranostic agent. Follow-up experiments are currently underway to validate the usefulness of cRGD-TMZ2-Cy72 in tumor-bearing animals.

\section{Acknowledgment}

This work was supported by the Deutsche Krebshilfe, D-53004 Bonn; Grant Number: 106335. The authors are thankful to Gabriele Müller, Biophysics of Macromolecules Department, DKFZ and Melanie Grundl, University of Applied Sciences, Mannheim for their encouragement.

\section{Conflict of Interest}

The authors have declared that no conflict of interest exists.

\section{References}

1. Pan D, Gambhir SS, Toyokuni T, et al. Rapid synthesis of a 5'-fluorinated oligodeoxy-nucleotide: a model antisense probe for use in imaging with positron emission tomography (PET). Bioorg Med Chem Lett. 1998; 8: 1317-20.

2. Chen X, Hou Y, Tohme M, et al. Pegylated Arg-Gly-Asp peptide: ${ }^{64} \mathrm{Cu}$ labeling and PET imaging of brain tumor $\alpha_{\mathrm{v}} \beta_{3}$-integrin expression. J Nucl Med. 2004; 45: 1776-83.

3. Beer AJ, Kessler H, Wester HJ, et al. PET Imaging of Integrin $\alpha_{v} \beta_{3}$ Expression. Theranostics. 2011; 1: 48-57.

4. Mühlhausen U, Komljenovic D, Bretschi M, et al. A novel PET tracer for the imaging of $\alpha_{v} \beta_{3}$ and $\alpha_{v} \beta_{5}$ integrins in experimental breast cancer bone metastases. Contrast Media Mol. Imag. 2011; in press.

5. Heckl S, Pipkorn R, Waldeck W, et al. Intracellular Visualization of Prostate Cancer Using Magnetic Resonance Imaging. Cancer Res. 2003; 63: 4766-72.

6. Mulder WJ, Strijkers GJ, van Tilborg GA, et al. Lipid-based nanoparticles for contrast-enhanced MRI and molecular imaging. NMR Biomed. 2006; 19: 142-64.

7. Zhang C, Jugold M, Woenne EC, et al. Specific targeting of tumor angiogenesis by RGD-conjugated ultrasmall superparamagnetic iron oxide particles using a clinical 1.5-T magnetic resonance scanner. Cancer Res. 2007; 67: 1555-62.

8. Tan M, Lu Z-R. Integrin targeted MR imaging. Theranostics. 2011; 1: 83-101.

9. Kaufmann BA, Lindner JR. Molecular imaging with targeted contrast ultrasound. Curr Opin Biotechnol. 2007; 18: 11-6.

10. Klibanov AL. Ultrasound molecular imaging with targeted microbubble contrast agents. J Nucl Cardiol. 2007; 14: 876-84.

11. Palmowski M, Huppert J, Ladewig G, et al. Molecular profiling of angiogenesis with targeted ultrasound imaging: early assessment of antiangiogenic therapy effects. Mol Cancer Ther. 2008; 7: 101-9.

12. Kiessling F, Gaetjens J, Palmowski M. Application of molecular ultrasound for imaging integrin expression. Theranostics. 2011; 1: 127-34.

13. Chen X, Conti PS, Moats RA. In vivo near-infrared fluorescence imaging of integrin $\alpha_{v} \beta_{3}$ in brain tumor xenografts. Cancer Res. 2004; 64: 8009-14.

14. Cheng $\mathrm{Z}, \mathrm{Wu} \mathrm{Y}, \mathrm{Xiong} \mathrm{Z}$, et al. Near-infrared fluorescent RGD peptides for optical imaging of integrin $\alpha_{\mathrm{v}} \beta_{3}$ expression in living mice. Bioconjug Chem. 2005; 16: 1433-41.

15. Cai W, Shin DW, Chen K, et al. Peptide-labeled near-infrared quantum dots for imaging tumor vasculature in living subjects. Nano Lett. 2006; 6: 669-76.

16. Ye $Y$, Chen $X$. Integrin targeting for tumor optical imaging. Theranostics. 2011; 1: 102-26.

17. Warner S. Diagnostics + Therapy $=$ Theranostics. The Scientist. 2004; 18: 38.

18. Xie J, Lee S, Chen X. Nanoparticle-based theranostic agents. Adv Drug Deliv Rev. 2010; 62: 1064-79.

19. Lammers T, Kiessling F, Hennink WE, et al. Nanotheranostics and image-guided drug delivery: current concepts and future directions. Mol Pharm. 2010; 7: 1899-912.

20. Chen X. Integrin Targeted Imaging and Therapy. Theranostics. 2011; 1: 28-9.

21. Braun K, Peschke P, Pipkorn R, et al. A biological transporter for the delivery of peptide nucleic acids (PNAs) to the nuclear compartment of living cells. J Mol Biol. 2002; 318: 237-43.

22. Pipkorn R, Debus J, Waldeck W, et al. Synthesis of functional peptides for therapy of HPV 18 positive hela cervix cancer cells. Biopolymers. 2003; 71: 409.

23. Braun K, von Brasch L, Pipkorn R, et al. BioShuttle-mediated plasmid transfer. Int J Med Sci. 2007; 4: 267-77.

24. Pipkorn R, Waldeck W, Didinger B, et al. Inverse-electron-demand Diels-Alder reaction as a highly efficient chemoselective ligation procedure: Synthesis and function of a BioShuttle for temozolomide transport into prostate cancer cells. J Pept Sci. 2009; 15: 235-41.

25. Wiessler $M$, Waldeck $W$, Kliem $C$, et al. The Diels-Alder-reaction with inverse-electron-demand, a very efficient versatile click-reaction concept for proper ligation of variable molecular partners. Int J Med Sci. 2009; 7: 19-28.

26. Pipkorn R, Wiessler M, Waldeck W, et al. TMZ-Bioshuttle, An Exemplary Drug Reformulation by Inverse Diels Alder Click Chemistry. Biopolymers. 2009; 92: 350-1.

27. Braun K, Wiessler M, Pipkorn R, et al. A cyclic-RGD-BioShuttle functionalized with TMZ by DARinv "Click Chemistry" targeted to $\alpha_{\mathrm{v}} \beta_{3}$ integrin for therapy. Int J Med Sci. 2010; 7: 326-39.

28. Wiessler, M, Kliem, C, Lorenz, P, Mueller, E, and Fleischhacker, H. EU Patent: Ligation reaction based on the Diels Alder Reaction with invers electron demand. 2006; [EP 06012 414.6].

29. Reppe W, Schlichting O, Klager K, et al. Cyclisierende Polymerisation von Acetylen I. Justus Liebigs Ann Chem. 1948; 560: 1-92.

30. Wiessler M, Waldeck W, Pipkorn R, et al. Extension of the PNA world by functionalized PNA monomers eligible candidates for inverse Diels Alder Click Chemistry. Int J Med Sci. 2010; 7: 213-23.

31. Atherton E, Sheppard RC. In: Undenfriend S, Meienhofer J, editors. The Peptides. Orlando: Academic Press. 1987:1-38.

32. Kubas H, Schäfer $M$, Bauder-Wüst $U$, et al. Multivalent cyclic RGD ligands: influence of linker lengths on receptor binding. Nucl Med Biol. 2010; 37: 885-91.

33. Weissleder R, Mahmood U. Molecular imaging. Radiology. 2001; 219: 316-33 
34. Lammers $T$, Aime $S$, Hennink WE, et al. Theranostic Nanomedicines. Acc Chem Res. 2011; 44: 1029-38.

35. Heckl S, Debus J, Jenne J, et al. CNN-Gd(3+) Enables Cell Nucleus Molecular Imaging of Prostate Cancer Cells: The Last 600 nm. Cancer Res. 2002; 62: 7018-24.

36. Nielsen PE, Egholm M, Berg RH, et al. Sequence-selective recognition of DNA by strand displacement with a thymine-substituted polyamide. Science. 1991; 254: 1497-500.

37. Egholm M, Buchardt O, Christensen L, et al. PNA hybridizes to complementary oligonucleotides obeying the Watson-Crick hydrogen-bonding rules [see comments]. Nature. 1993; 365: 566-8.

38. Demidov VV, Potaman VN, Frank Kamenetskii MD, et al. Stability of peptide nucleic acids in human serum and cellular extracts. Biochem Pharmacol. 1994; 48: 1310-3.

39. Nielsen PE. Applications of peptide nucleic acids. Curr Opin Biotechnol. 1999; 10: 71-5.

40. Nielsen PE. Peptide nucleic acids and the origin of life. Chem Biodivers. 2007; 4: 1996-2002.

41. Fletcher MP, Seligmann BE. Monitoring human neutrophil granule secretion by flow cytometry: secretion and membrane potential changes assessed by light scatter and a fluorescent probe of membrane potential. J Leukoc Biol. 1985; 37: 431-47.

42. Xiao L, Zhang Y, Liu Z, et al. Synthesis of the Cyanine 7 labeled neutrophil-specific agents for noninvasive near infrared fluorescence imaging. Bioorg Med Chem Lett. 2010; 20: 3515-7. 